\title{
Finding the Peak Age of an Indian Woman Victim of Breast Cancer using CETD Matrix
}

\author{
A. Victor Devadoss, PhD \\ Department of Mathematics, \\ Loyola College, Chennai
}

\author{
J. Janet Sheeba \\ Department of Mathematics, \\ Loyola College, Chennai
}

\author{
M. Albert William, PhD \\ Department of Mathematics, \\ Loyola College, Chennai
}

\begin{abstract}
Cancer occurs as a result of mutations, or abnormal changes, in the genes responsible for regulating the growth of cells and keeping them healthy. The genes are in each cell's nucleus, which acts as the "control room" of each cell. Normally, the cells in our bodies replace themselves through an orderly process of cell growth: healthy new cells take over as old ones die out. But over time, mutations can "turn on" certain genes and "turn off" others in a cell. That changed cell gains the ability to keep dividing without control or order, producing more cells just like it and forming a tumor. The breast has developed from cells in the breast due to a malignant tumor. Usually breast cancer either begins in the cells of the lobules, which are the milkproducing glands, or the ducts, the passages that drain milk from the lobules to the nipple. Less commonly, breast cancer can begin in the stromal tissues, which include the fatty and fibrous connective tissues of the breast. Breast cancer is always caused by a genetic abnormality. However, only $5-10 \%$ of cancers are due to an abnormality inherited from your mother or father. About $90 \%$ of breast cancers are due to genetic abnormalities that happen as a result of the aging process and the "wear and tear" of life in general. According to Hindu September 30, 2009, The Experts project breast cancer to strike approximately 2.5 lakh women in India by 2015. "Breast cancer has overtaken cervical cancer to become the leading cause of cancerrelated mortality among women living in metropolitan cities," says the Indian Council of Medical Research which places incidence of the disease at 30 to 33 per 1,00,000 women in urban India. Cancer rates could further increase by 50 per cent to 15 million new cases in the year 2020, according to the World Cancer Report. The report also reveals the developing world is expected to account for more than half of all cancer cases in the world by 2020 . According to B.Niranjan Naik of Dharamshila hospital, a cancer specialist hospital, almost 75,000 new cases of breast cancer are detected in India every year. The WHO is also warning that Asia's annual death toll from cancer, currently at about 4 million, could reach 6.4 million by 2030 if current trends continue and the incidence of new cases in Asia is rising by around 60 per cent in some parts. Dr. Col. C. S. Pant, Vice Chairperson for the Forum for Breast Cancer Protection, "The rise is particularly affecting younger women between 30 and 40 years. Unlike in the West where typically women after 50 years get early stage disease, breast cancer in Asian women occurs at a younger age and is usually presented and diagnosed at a later stage."
\end{abstract}

In this paper an attempt is made to find out the peak age of a women getting breast cancer in Chennai by the method of unsupervised questioner. We collected the data from 100 patients of Adyar Cancer Institute, Chennai. The following are identified as the symptoms of Breast Cancer namely breast pain, rash/itching, swelling, skin retraction, nipple discharge, lump, left/right breast large in size. First section gives the details about Breast Cancer. Section two deals with the concept of RTD matrix. In section three the data being analyzed using CETD matrix. In section four conclusions and suggestions are derived.

\section{Keywords}

Cancer, Breast cancer, Breast pain, Symptoms, Rash/itching, Swelling, Skin retraction, Nipple discharge, Lump, ATD matrix, RTD matrix and CETD Matrix.

\section{INTRODUCTION}

Breast cancer is a malignant tumor that starts in the cells of the breast. A malignant tumor is a group of cancer cells that can grow into (invade) surrounding tissues or spread (metastasize) to distant areas of the body. The disease occurs almost entirely in women, but men can get it, too(1). The incidence of breast cancer is rising in India and is now the second most common cancer diagnosed in women after cervical cancer. It is estimated that in 2008 there were 115,251 new cases of breast cancer with an age standardized incidence rate of 22.9 per 100,000 . It is estimated that by 2030 the number of new cases of breast cancer in India will reach just fewer than 200,000 per year. According to study of Farley et al. (2000), Cancer of the breast in women is a major health burden worldwide. It is a growing problem in both high-resource and low-resource settings, and is responsible for over one million of the estimated 10 million neoplasms diagnosed worldwide each year in both sexes. It is also the primary cause of cancer death among women globally, responsible for about 375,000 deaths in the year 2000(2). It is very evident from the various statistics, that the incidence of breast cancer is rapidly rising, amounting to a significant percentage of all cancers in women. Breast cancer is the commonest cancer in urban areas in India and accounts for about $25 \%$ to $33 \%$ of all cancers in women. If these percentages are converted into actual numbers, the numbers are very high! Combine this with the fact, that over $50 \%$ breast cancer patients in India present in stages 3 and 4, which will definitely, impact the survival. 


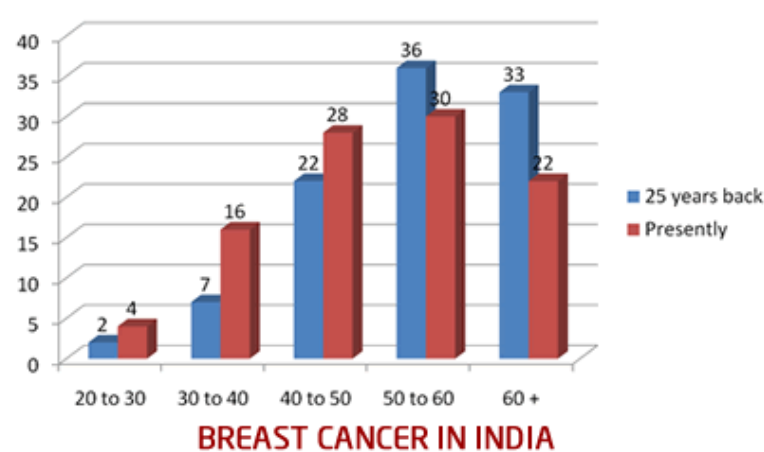

In India, the average age of developing a breast cancer has undergone a significant shift over last few decades. 25 years back, out of every 100 breast cancer patients, $2 \%$ were in 20 to 30 years age group, $7 \%$ were in 30 to 40 and so on. $69 \%$ of the patients were above 50 years of age. Presently, $4 \%$ are in 20 to 30 yrs age group, $16 \%$ are in 30 to $40,28 \%$ are in 40 to 50 age group. So, almost $48 \%$ patients are below 50. An increasing numbers of patients are in the 25 to 40 years of age, and this definitely is a very disturbing trend (3). According to study of Sinha (2011), the maiden global analysis that factored the trend over the past three decades shows that the number of new breast cancer cases diagnosed worldwide has increased dramatically from about 6.4 lakhs in 1980 to 16 lakhs in 2010(2). In our study an attempt is made to find the peak age of an Indian woman in Chennai becoming the victim of breast cancer.

\section{THE CONCEPT OF RTD MATRIX: 2.1. Average Time Dependent (ATD) matrix}

Raw data is transformed into a raw time dependent data matrix by taking along the rows the details of the age group and along the columns the number of occurrences of different symptoms. We make it into the Average Time Dependent Data (ATD) matrix $\left(a_{i j}\right)$ by dividing each entry of the raw data matrix by the number of years i.e., the time period. This matrix represents a data, which is totally uniform. At the third stage we find the average and Standard Deviation (S.D) of every column in the ATD matrix.

\subsection{Refined Time Dependent (RTD) matrix:}

Using the average $\mu_{j}$ of each $j^{\text {th }}$ column and $\sigma_{j}$ the S.D of the each $j^{\text {th }}$ column we chose a parameter $\alpha$ from the interval $[0,1]$ and form the interval $[0,1]$ and form the Refined time dependent Matrix (RTD matrix), Using the formula

$$
\begin{aligned}
& a_{i j} \leq\left(\mu_{j}-\alpha * \sigma_{j}\right) \text { then } e_{i j}=-1 \text { else } \\
& \qquad \text { if } a_{i j} \in\left(\mu_{j}-\alpha * \sigma_{j}, \mu_{j}+\alpha * \sigma_{j}\right) \text { then }
\end{aligned}
$$

$e_{i j}=0$ else

$$
\text { if } a_{i j} \geq\left(\mu_{j}+\alpha * \sigma_{j}\right) \text { then } e_{i j}=1 \text {. }
$$

We redefine the ATD matrix into the Refined time dependent fuzzy matrix for here the entries are $=0$ or 1 . Now the row sum of this matrix gives the maximum age group.

\subsection{Combined Effective Time Dependent Data (CETD) matrix}

We also combine the above RTD matrices by varying the $\alpha \in[0,1]$ Dependent Data (CETD) matrix. The row sum is obtained for CETD matrix and conclusions are derived based on the row sums. All these are represented by graphs and graphs play a vital role in exhibiting the data by the simplest means, which can be even understood by a layman $(4,5)$.

\section{DESCRIPTION OF THE PROBLEM}

Cancer occurs as a result of mutations, or abnormal changes, in the genes responsible for regulating the growth of cells and keeping them healthy. The term "breast cancer" refers to a malignant tumor that has developed from cells in the breast. Usually breast cancer either begins in the cells of the lobules, which are the milk-producing glands, or the ducts, the passages that drain milk from the lobules to the nipple. Less commonly, breast cancer can begin in the stromal tissues, which include the fatty and fibrous connective tissues of the breast. Breast cancer is always caused by a genetic abnormality $(6,7)$. However, only $5-10 \%$ of cancers are due to an abnormality inherited from the mother or father. About $90 \%$ of breast cancers are due to genetic abnormalities that happen as a result of the aging process and the "wear and tear" of life in general. The number of breast cancer cases in India is about 100,000 women each year and there will be approximately 2,50,000 new cases of breast cancer in India by 2015, says Indian Council of Medical Research (ICMR).Cancer rates could further increase by 50 per cent to 15 million new cases in the year 2020, according to the World Cancer Report, the most comprehensive global examination of the disease to date.

Age is the single most important risk factor for breast cancer. The study conducted by Karabi Dattan in eight year time period, shows major patients were in the 40-49 year old age group in $1997-2004,29.69 \%$ in 1997 to $32.04 \%$ in 2002 and finally to $27.85 \%$ in 2004 and the next most commonly affected age group was 50-59 year age group which varies from $22.80 \%$ in 1997 to $24.60 \%$ in 2002 and ultimately to $24.43 \%$ in 2004 . Hence the effectiveness of the treatment and the age factor reflects on the aggressiveness of the disease. Due to the poor patient follow up an inadequate system of registration of death the survival of breast cancer patient is very negligible. So, we have taken a research study using fuzzy logic, in particular using CETD matrix how the age factor and the different stages of breast cancer march together towards the death point of the breast cancer patient. 
A landmark analysis of cancer cases among women in Delhi, Mumbai, Chennai and Bangalore from 1982 to 2005, conducted by the Indian Council of Medical Research (ICMR) revealed that the incidence of breast cancer nearly doubled in metropolitan cities(8). If the cancer patient understands the age factor and the different stages of breast cancer there can be a chance of life for every passing year. This will reduce the death rate of cancer patient gradually. So, better pattern of care in terms of diagnosis and treatment could be reflected in the survival of breast cancer.

Using an unsupervised method a data is collected from the 100 breast cancer patient. We have interviewed 100 women breast cancer patients who visited in Adyar cancer institute in Chennai. The following symptoms of breast cancer are breast pain, rash/itching, swelling, skin retraction, nipple discharge, lump, left/right breast large in size are identified. We analyze these problems using fuzzy matrix, we call the RTD Matrix is fuzzified by the entries from the set $\{-1,0,1\}$.

In this paper we will discuss symptoms of breast cancer, which are taken as the columns of the initial row data matrix the age group in years, $12-20,21-35,36-45,46-60 \& 61-80$. The estimation of the maximum age group is five-stage process. In the first stage we give the matrix representation of the raw data. The $3 \times 8$ matrix is not uniform i.e. the number of individual years in each interval may not be the same. So in the second stage, in order to obtain an unbiased uniform effect on each and every data so collected, transform this initial matrix into an Average Time Dependent Data (ATD) matrix. To make the calculations easier and simpler, in the third stage using the simple average techniques convert the above average time dependent data matrix in to a matrix with entries $e_{i j} \in\{-1,0,1\}$

Dependent Data Matrix (RTD Matrix) or as the fuzzy matrix.

The value of $e_{i j}$ corresponding to each entry is determined in a special way. At the fourth stage using the fuzzy matrices we obtain the Combined Effect Time Dependent Data Matrix (CETD Matrix), which gives the cumulative effect of all these entries. In the final stage we obtain the row sums of the CETD matrix. The graph of the RTD matrix and CETD matrix are given.

\subsection{Estimation of maximum age group of Breast Cancer patients by using $5 \times 8$ matrices:}

In this section we take eight attributes related to symptoms of Breast Cancer

$S_{1}$ - breast pain, $S_{2}$ - Rash/itching, $S_{3}$ - Swelling, $S_{4}$ - Skin retraction, $S_{5}$ - Nipple discharge, $S_{6}$ - Lump, $S_{7}$-Left/right breast large in size $\& S_{8}$ - Same size to the CETD model.

Initial raw data matrix of order 5 x 8

\begin{tabular}{|l|l|l|l|l|l|l|l|l|}
\hline years & $S_{1}$ & $S_{2}$ & $S_{3}$ & $S_{4}$ & $S_{5}$ & $S_{6}$ & $S_{7}$ & $S_{8}$ \\
\hline $12-20$ & 2 & 8 & 6 & 12 & 9 & 10 & 9 & 2 \\
\hline $21-35$ & 18 & 14 & 18 & 16 & 19 & 18 & 21 & 14 \\
\hline $36-45$ & 16 & 18 & 28 & 14 & 29 & 28 & 20 & 24 \\
\hline $46-60$ & 36 & 14 & 27 & 31 & 32 & 52 & 28 & 24 \\
\hline $61-80$ & 4 & 1 & 2 & 4 & 2 & 8 & 3 & 8 \\
\hline
\end{tabular}

The ATD matrix of order $5 \times 8$

\begin{tabular}{|l|l|l|l|l|l|l|l|l|}
\hline years & $S_{1}$ & $S_{2}$ & $S_{3}$ & $S_{4}$ & $S_{5}$ & $S_{6}$ & $S_{7}$ & $S_{8}$ \\
\hline $12-20$ & 0.22 & 0.88 & 0.66 & 1.33 & 1 & 1.11 & 1 & 0.22 \\
\hline $21-35$ & 1.2 & 0.93 & 1.2 & 1.06 & 1.26 & 1.2 & 1.4 & 0.93 \\
\hline $36-45$ & 1.6 & 1.8 & 2.8 & 1.4 & 2.9 & 2.8 & 2 & 2.4 \\
\hline $46-60$ & 2.4 & 0.93 & 1.8 & 2.06 & 2.13 & 3.46 & 1.86 & 1.6 \\
\hline $61-80$ & 0.2 & 0.05 & 0.1 & 0.2 & 0.1 & 0.4 & 0.15 & 0.4 \\
\hline
\end{tabular}

The Average and Standard deviation of the above ATD Matrix

\begin{tabular}{|l|l|l|l|l|l|l|l|l|}
\hline Average & 1.12 & 0.91 & 1.31 & 1.21 & 1.47 & 1.79 & 1.28 & 1.11 \\
\hline S .D & 0.93 & 0.61 & 1.04 & 0.67 & 1.07 & 1.27 & 0.74 & 0.89 \\
\hline
\end{tabular}


The RTD matrix for $\alpha=0.27$

$\left[\begin{array}{cccccccc}-1 & 0 & -1 & 0 & -1 & -1 & -1 & -1 \\ 0 & 0 & 0 & 0 & 0 & -1 & 0 & 0 \\ 1 & 1 & 1 & 1 & 1 & 1 & 1 & 1 \\ 1 & 0 & 1 & 1 & 1 & 1 & 1 & 1 \\ -1 & -1 & -1 & -1 & -1 & -1 & -1 & -1\end{array}\right]$

The RTD matrix for $\alpha=0.43$

$\left[\begin{array}{cccccccc}-1 & 0 & -1 & 0 & -1 & -1 & 0 & -1 \\ 0 & 0 & 0 & 0 & 0 & -1 & 0 & 0 \\ 1 & 1 & 1 & 0 & 1 & 1 & 1 & 1 \\ 1 & 0 & 1 & 1 & 1 & 1 & 1 & 1 \\ -1 & -1 & -1 & -1 & -1 & -1 & -1 & -1\end{array}\right]$

The RTD matrix for $\alpha=0.62$

$\left[\begin{array}{cccccccc}-1 & 0 & -1 & 0 & 0 & 0 & 0 & -1 \\ 0 & 0 & 0 & 0 & 0 & 0 & 0 & 0 \\ 0 & 1 & 1 & 0 & 1 & 1 & 1 & 1 \\ 1 & 0 & 0 & 1 & 0 & 1 & 1 & 0 \\ -1 & -1 & -1 & -1 & -1 & -1 & -1 & -1\end{array}\right]$

The RTD matrix for $\alpha=0.78$

$\left[\begin{array}{cccccccc}-1 & 0 & 0 & 0 & 0 & 0 & 0 & -1 \\ 0 & 0 & 0 & 0 & 0 & 0 & 0 & 0 \\ 0 & 1 & 1 & 0 & 1 & 1 & 1 & 1 \\ 1 & 0 & 0 & 1 & 0 & 1 & 1 & 0 \\ -1 & -1 & -1 & -1 & -1 & -1 & -1 & -1\end{array}\right]$

The row sum matrix

$$
\left[\begin{array}{c}
-6 \\
-1 \\
8 \\
7 \\
-8
\end{array}\right]
$$

The row sum matrix

$$
\left[\begin{array}{c}
-5 \\
-1 \\
7 \\
7 \\
-8
\end{array}\right]
$$

The row sum matrix

$$
\left[\begin{array}{c}
-3 \\
0 \\
6 \\
4 \\
-8
\end{array}\right]
$$

The row sum matrix

$$
\left[\begin{array}{c}
-2 \\
0 \\
6 \\
4 \\
-8
\end{array}\right]
$$

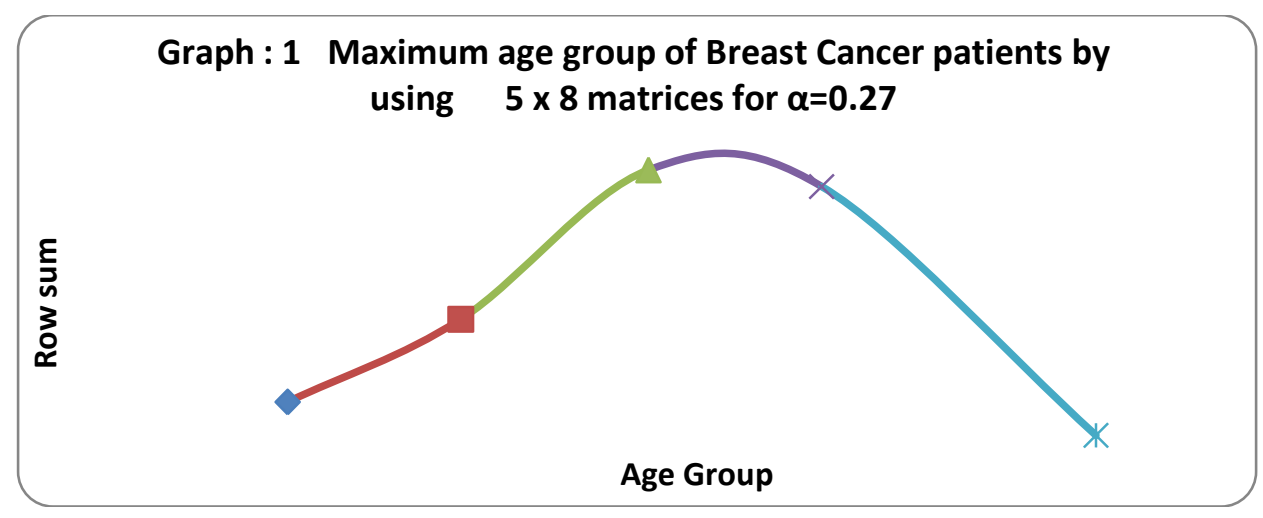



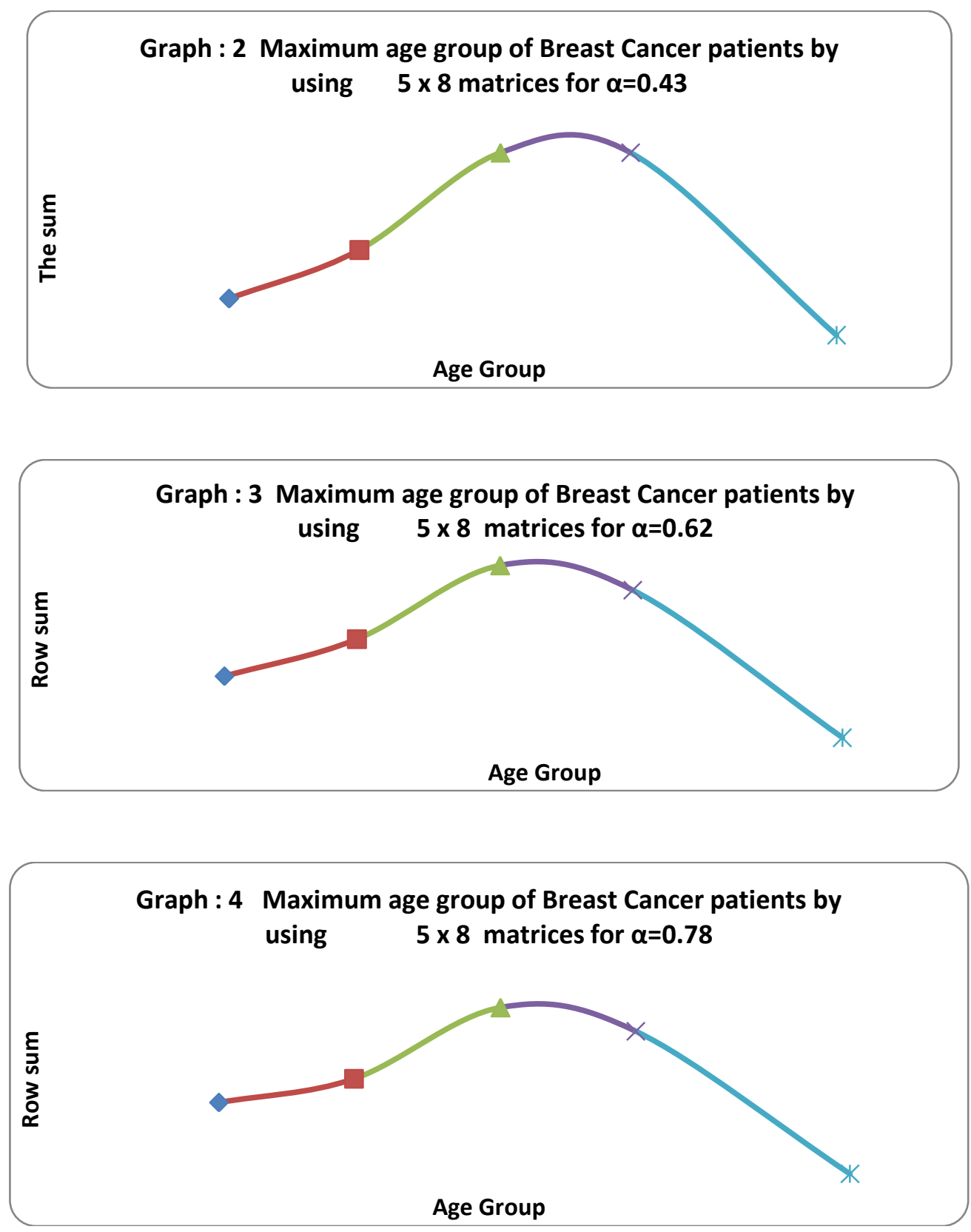

The CETD matrix
$\left[\begin{array}{cccccccc}-4 & 0 & -3 & 0 & -2 & -2 & -1 & -4 \\ 0 & 0 & 0 & 0 & 0 & -2 & 0 & 0 \\ 2 & 4 & 4 & 1 & 4 & 4 & 4 & 4 \\ 4 & 0 & 2 & 4 & 2 & 4 & 4 & 2 \\ -4 & -4 & -4 & -4 & -4 & -4 & -4 & -4\end{array}\right]$

The row sum matrix

$\left[\begin{array}{c}-16 \\ -2 \\ 27 \\ 22 \\ -32\end{array}\right]$




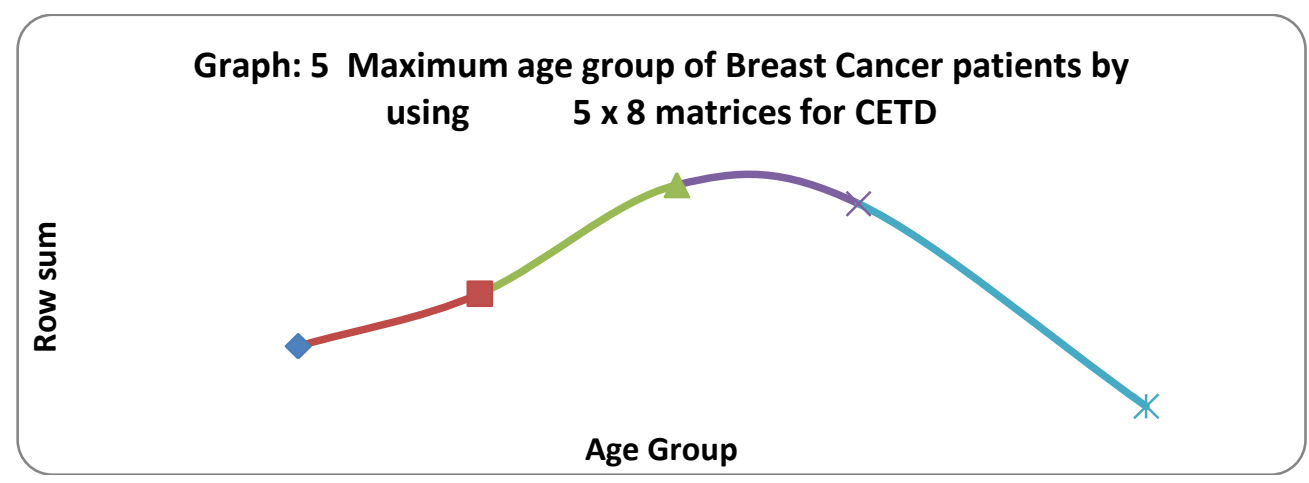

From the graph the Breast Cancer starts at the age of 18 . The peak age of women having Breast Cancer is 48 . The peak period is 48 to 51.

\section{CONCLUSION}

There are three different stages of breast cancer on the basis of development taking in the breast viz.

- $\quad$ Tumor extent (T)

- $\quad$ Nodal spread (N)

- $\quad$ Metastatic (Distant) spread (M)

Using the study of CETD matrix age group of the stage of breast cancer is determined and the peak age of the women.

\section{Tumor extent (T):}

To stage the ' $\mathrm{T}$ ' part, the surgeon will assess the size of the lump in the breast. Apart from the size, he has to see, whether the skin overlying the tumor is involved, whether the nipple is retracted, whether the tumor is densely 'adherent' to the underlying chest wall. After understanding all these factors, the ' $\mathrm{T}$ ' part is staged. According to the latest classification by the AJCC (7th ed), the T classification is as follows:

- Between 18-25, T1: Tumour less than $2 \mathrm{~cm}$ in greatest dimension.

- $\quad$ Between 26-48, T2: Tumour more than $2 \mathrm{~cm}$ but less than $5 \mathrm{~cm}$ in greatest dimension.

- Between 48 -50, T3: Tumour more than $5 \mathrm{~cm}$ in greatest dimension.

- Between 51- 70, T4: Tumor of any size with direct extension to the chest wall and/or to the skin (ulceration or skin nodules)

T1 to T3 are 'operable' lesions. A tumour will keep on growing and ultimately involve the skin or underlying chest wall, and it then becomes T4 which is defined as 'locally advanced cancer', and is not good, since the moment the tumour involves overlying skin or underlying chest wall, it's propensity to recur back after treatment, and propensity to spread to distant sites is much more. Once a cancer is 'locally advanced', it is usually not amenable to direct surgery and will need to be treated by a chemotherapy first to with an aim to make it operable.

\section{Nodal spread (N):}

To stage the ' $\mathrm{N}$ ' part, the surgeon will assess the spread of the cancer in the axilla, by inserting his fingers in the axilla and feeling for any enlarged lymph nodes in the axilla in all directions and also by 'insinuating' fingers deep in the arm pit. The gives a fair idea of the 'nodal' spread of the cancer. After assessment, the nodal spread (the $\mathrm{N}$ part) is classified as follows:

- Between 18- 28 , N0: There are no enlarged 'nodes' in the axilla

- Between 26-48, N1: Nodes in the axilla are enlarged and can be felt, but they are mobile when the surgeon attempts to hold them between his thumb and fingers and move them in all directions.

- Between $48-50, \mathrm{~N} 2$ : Nodes in the axilla are enlarged and can be felt by the surgeon, and they are attached to one another ('matted') or to surrounding structures.

- Between 51- 70, N3: Enlarged nodes can be felt either below or above the collar bone on the same side, irrespective of nodes in the axilla.

Here again, as long as the nodes are mobile (N1), they can be operated upon primarily. But once the nodes become matted (N2) to one another or to surrounding structures, they come under definition of 'locally advanced breast cancer', and will need a prior chemotherapy followed by surgery (after reassessment of response).

\section{Distant spread (M):}

The distant spread is assessed by certain investigations like an ultrasonography of the abdomen to see for liver and other organs, an X ray of the chest (or if need be, a CT Scan) to see for lung involvement or collection of fluid in the chest, a bone scan to see for spread to bones, etc.

- Between 18 - 45, M0 There is no evidence of any distant spread of the cancer.

- Between 46 - 70, M1 Cancer has spread beyond the breast to any distant site, like the lung, brain, liver, bones etc.

Once the $\mathrm{T}, \mathrm{N}$ and $\mathrm{M}$ status is known, the final clinical staging is done to decide upon further treatment. To note, this is only a clinical staging. Once surgery is done, the specimen 
is assessed in histopathology and the final $\mathrm{T}$ and $\mathrm{N}$ staging is done, which is the pathological staging, on which further treatment is based.

\section{SUGGESTIONS}

1. Every person has cancer cells in the body. These cancer cells do not show up in the standard

tests until they have multiplied to a few billion. When doctors tell cancer patients that there are no more cancer cells in their bodies after treatment, it just tests are unable to detect the cancer cells because they have not reached the detectable size.

2. Cancer cells occur between 6 to more than 10 times in a person's life time.

3 . When the person's immune system is strong the cancer cells will be destroyed and prevented

from multiplying and forming tumors.

4. When a person has cancer it indicates the person has multiple nutritional deficiencies. These could be due to genetic, environmental, food and lifestyle factors. To overcome the multiple nutritional deficiencies, changing diet and including supplements will strengthen the immune system.

5. Initial treatment with chemotherapy and radiation will often reduce tumor size. However Prolonged use of chemotherapy and radiation do not result in more tumor destruction.

6. An effective way to battle cancer is to STARVE the cancer cells by not feeding it with foods it needs to multiple.

a. Sugar is a cancer-feeder. By cutting off sugar it cuts off one important foods supply to the cancer cells.

b. Milk causes the body to produce mucus, especially in the gastro-intestinal tract. Cancer feeds on mucus. By cutting off milk and substituting with unsweetened soy milk, cancer will starved.

c. Cancer cells thrive in an acid environment. A meat-based diet is acidic and it is best to eat fish, and a little chicken rather than beef or pork. Meat also contains livestock antibiotics, growth hormones and parasites, which are all harmful, especially to people with cancer.

d. A diet made of $80 \%$ fresh vegetables and juice, whole grains, seeds, nuts and a little fruits help put the body into an alkaline environment. About $20 \%$ can be from cooked food including beans. Fresh vegetable juices provide live enzymes that are easily absorbed and reach down to cellular levels within 15 minutes to nourish and enhance growth of healthy cells.

To obtain live enzymes for building healthy -cells try and drink fresh vegetable juice (most vegetables including bean sprouts) and eat some raw vegetables 2 or 3 times a day. Enzymes are destroyed at temperatures of 104 degrees F (40 degrees C)

e. Avoid coffee, tea, and chocolate, which have high caffeine. Green tea is better alternative and

has cancer-fighting properties. Water-best to drink purified water, or filtered, to avoid known toxins and heavy metals in tap water. Distilled water is acidic, avoid it.
7. Meat protein is difficult to digest and requires a lot of digestive enzymes. Undigested meat remaining in the intestines will become purified and leads to more toxic buildup.

8. Cancer cell walls have a tough protein covering. By refraining from or eating less meat it frees more enzymes to attack the protein walls of cancer cells and allows the body's killer cells to destroy the cancer cells.

9. Some supplements build up the immune system (IP6, Flor-essence, Essiac, anti-oxidants, vitamins, minerals, EFAs etc) to enable the body's own killer cells to destroy cancer cells. Other supplements like vitamin E are known to cause apoptosis, or programmed cell death, the body's normal method of disposing of damaged. Unwanted or unneeded cells.

10 Cancer is the disease of mind, body and spirit. A proactive and positive spirit will help the cancer warrior be a survivor. Anger, unforgiving and bitterness put the body into a stressful and acidic environment. Learn to have a loving and forgiving spirit. Learn to relax and enjoy life.

11. Cancer cells cannot thrive in an oxygenated environment. Exercising daily, and deep breathing help to get more oxygen down to the cellular level. Oxygen therapy is another means to destroy cancer cells.

\section{REFERENCES}

[1] http://www.cancer.org/cancer/breastcancerinmen/detaile dguide/breast-cancer-in-men-what-is- breast-cancer-inmen

[2] Breast Cancer Scenario in a Regional Cancer Centre in Eastern India over Eight Years - Still a Major Public Health Problem Karabi Datta*, Maitrayee Choudhuri, Subhas Guha, Jaydip BiswasAsian Pacific Journal of Cancer Prevention, Vol 13, $2012 \quad 809$ DOI:http://dx.doi.org/10.7314/APJCP.2012.13.3.809 Eight Year Data for Breast Cancer in Kolkata, India

[3] http://www.breastcancerindia.net/bc/statistics/trends.htm.

[4] Vasantha Kandasamy. W.B, Elumalai, Victor Devadass and Mary John "Application of CETD Matrix Technique to study the Social and Psychological problems faced by RAG pickers (2007).

[5] Vasantha Kandasamy. W.B, Mary John. M and

[6] Kanagamuthu. T(2002) "Study of Social Interaction and Women Empowerment Relative to HIV/AIDS".

[7] Bart Kosko, "Neural Networks and Fuzzy Systems",

[8] Prentice Hall of India Private Limited, (1967).

[9] http://www.medicinenet.com/breast_cancer/article.htm.

[10] October 2011 Current Affairs, Report | Survey, News Capsule, 2011 Current Affairs 